\title{
Bone Marrow Cytomorphology And Clinico-Hematological Profile of Metastatic Solid Tumors: A 5-Year Audit from A Tertiary Care Centre in Northern India
}

\author{
Vijay Kumar, Rohan Sardana, Manjari Kishore*, Sadhna Marwah, A.S. Nigam and Minakshi Bhardwaj \\ Dept of Pathology, PGIMER, Dr RML Hospital, New Delhi. India
}

\begin{abstract}
Introduction: Metastasis to bone marrow by solid malignancies is a rare event and it upgrades the tumor thereby worsening the prognosis. This is caused by an impaired response to therapy and other manifestation resulting from decreased hematopoiesis. The common malignancies to metastasize in male and female are carcinoma prostate and carcinoma breast, respectively. It is noted that metastasis from small round cell tumor (SRCT) predominates in children. Here, we present a series of cases of bone marrow metastasis by solid organ malignancies over a period of 5 years.
\end{abstract}

Objectives: The aim of this study was to investigate the clinical and hematological characteristics of patients with BM metastases of solid tumors diagnosed by bone marrow (BM) aspiration.

Materials \& Methods: In this study, we reviewed 3520 bone marrow aspirates in our hospital from January 2013 to December 2017. Patients diagnosed with solid malignancies metastasizing to bone marrow were selected.

Results: We found a total of 26 cases of solid malignancies with metastasis to bone marrow. The age of the patients ranged from $2-88$ years (mean age of 46 years). Most of the patients were adults (19/26), of which $77.7 \%$ were males. Seven of the 26 patients were in the pediatric age group, with an age range of 2-11 years. Various malignancies metastasizing to marrow were evaluated and clinical and hematological correlation was done. The common presenting sign \& symptoms were fever, anorexia and bodyache and organomegaly. Anemia was the most common hematological finding at the time of admission. Carcinoma prostate was the most common metastatic tumor in adults and small round cell tumor in children.

Conclusion: BM is one of the important and infrequent sites of metastasis of solid tumors. Detection of bone marrow metastasis has significant clinical implication with impact on therapeutic decisions and is associated with poor prognosis. Aspiration of the BM provides an easy and quick way of detecting involvement, however, biopsy with immunohistochemical and/or molecular techniques may be needed in case of microscopical tumor burden.

Keywords: Bone Marrow, Immunohistochemistry, Metastasis, Solid Malignancies.

\section{Introduction}

Metastasis to bone marrow (BM) by non-haematological malignancies is a rare event and is often associated with a worsened prognosis. ${ }^{[1]}$ A reduction in overall survival, impaired response to therapy and multiple other manifestations resulting from decreased hematopoiesis are noted. Carcinoma prostate and carcinoma breast are the most common malignancies to metastasize in males and females respectively. ${ }^{[1-3]}$ Metastasis from lungs, gastrointestinaltract and ewing's sarcoma account for majority of cases. Neuroblastoma, rhabdomyosarcoma, ewing's sarcoma and other small round cell tumors (SRCT) metastasize to the $\mathrm{BM}$ in children. ${ }^{[1-4]}$ The patients with $\mathrm{BM}$ involvement may or may not have any presenting complaint and hence a keen clinical observation is imperative in such cases. Here, we present a series of cases of BM metastasis by solid organ malignancies over a period of 5 years.

\section{Materials and Methods}

This study was conducted retrospectively looking at archival records and slides over a period of 5 years. Out of 3520 cases so reviewed, 26 cases showed metastatic malignancies. Bone marrows were sent for either refractory anemia or pancytopenia or for staging of tumor. The age, sex, chief complaints, physical examination, lymph node status, relevant biochemical findings, peripheral blood smear findings were retrieved and tabulated.

WHO guidelines for defining anemia, thrombocytopenia and leucopenia were followed. The radiological findings [X-ray/ or Computed Tomography (CT) findings] wherever available were also noted. Biochemical investigations mostly included serum calcium, lactic acid dehydrogenase (LDH), alkaline phosphatase (ALP), and serum tumor markers like PSA level. 
Bone marrow aspirations and trephine biopsies were performed on posterior superior iliac spine using Jamshidi needle. Bone marrow smears were stained with Giemsa and peripheral blood smear \& reticulocyte count were also performed in all cases. Bone marrow biopsy was not available for all patients and whenever available were stained with hematoxylin and eosin stain. Reticulin and Masson's trichome were done wherever fibrosis was suspected. Imunohistochemical analysis was also performed wherever possible.

\section{Results}

On proper evaluation of bone marrow aspirate smears \& biopsies, 26 cases were found to be positive for metastatic deposits over a period of 5 years. Out of the 3520 bone marrows, cases with evidence of multiple myeloma, nonhodgkin lymphoma, leukemia infiltration and hodgkin lymphoma were excluded from the study.

The age of the patients ranged from 2-88 years (mean age of 46 years). Most of the patients were adults (19/25), of which $77.7 \%$ were males. Seven of the 26 patients $(26.92 \%)$ were in the pediatric age group, with an age range of 2-11 years. Among children, boys had a slightly higher incidence of $57.2 \%$.

The most common complaints were fever, anorexia and bodyache. Physical examination revealed pallor and organomegaly in 10 of 26 cases $(38.46 \%)$ of the cases. The radiological findings were available in 8 cases. At the time of diagnosis, anemia was the most common hematological finding $(65 \%)$. Pancytopenia was present in 8 patients (31\%) and leukoerythroblastic picture was present in 4 cases only (15.4\%). The peripheral blood findings have been tabulated as follows in Table no.1.

The biochemical findings included most commonly raised ALP and LDH. Serum bilirubin was raised only in a few cases ( 3 of 26 cases, 11.54\%). The biochemical findings have been summarized in Table 2 .

The bone marrow aspirate smears were cellular in $52 \%$ of the cases and hemodilute in the remaining. One patient had only bone marrow biopsy (no aspirate smear) sent for examination. Megakaryocytes were not seen in two cases $(8 \%)$; however, were occasionally noted in $52 \%$ of the cases. Out of the 26 cases, we had received 25 bone marrow aspirate slides. In 11 of the cases, bone marrow biopsy was received out of which 2 were inadequate for any opinion. Reticulin stain performed on bone marrow biopsy sections showed fibrosis in 4 of 11 cases $(36.36 \%)$.

All paediatric cases had metastasis from small round cell tumor (SRCT). Of these 7 cases of small round cell tumor, neuroblastoma (4/7) [Figure 1A-D] was the most common solid tumor from where metastasis had been documented in these children.

Carcinoma prostate was the most common malignancy to be diagnosed [Figure 2A-D] in adults followed by metastasis from breast carcinoma [Figure 3A-B] \& hepatic/ gall bladder [Figure 3C-D]. Immunohistochemistry (IHC) was applied in selected cases to confirm the epithelial malignancy and the small round cell tumor. Table 3 summarises various solid malignancies with evidence of bone marrow metastasis.

Table 1: Table showing the peripheral smear findings in all the patients with bone marrow metastasis, ( $n=26)$ :

\begin{tabular}{|c|c|}
\hline Peripheral blood picture & No. of cases \\
\hline Normocytic normochromic & 6 \\
\hline Pancytopenia & 8 \\
\hline Bicytopenia & 4 \\
\hline Leukoerythroblastic & 4 \\
\hline Microcytic hypochromic & 2 \\
\hline Macrocytic normochromic & 1 \\
\hline Thrombocytopenia & 1 \\
\hline Total no of cases & $\mathbf{2 6}$ \\
\hline
\end{tabular}

Table 2: Table depicting the major biochemical alterations noted in the patients.

\begin{tabular}{|c|c|c|}
\hline S. No. & Biochemical parameters & No. of cases \\
\hline 1 & Elevated LDH levels & 6 \\
\hline 2 & Elevated Alkaline phosphatise & 3 \\
\hline 3 & Deranged KFT & 3 \\
\hline 4 & Deranged LFT & 2 \\
\hline 5 & Hypertriglycerides & 6 \\
\hline 6 & Raised PSA levels & 2 \\
\hline 7 & Raised CPK levels & 3 \\
\hline
\end{tabular}


Table 3: Primary diagnoses of the patients with metastasis to bone marrow, $(n=26)$ :

\begin{tabular}{|c|c|c|}
\hline S. No & Diagnoses & No of cases \\
\hline 1 & Small round cell tumor & 7 \\
\hline 2 & Carcinoma prostate & 1 \\
\hline 3 & Carcinoma breast & 1 \\
\hline 4 & Liver/gall bladder carcinoma & 2 \\
\hline 5 & Adenocarcinoma colon & 7 \\
\hline 6 & Unknown primary with evidence of malignant \\
\hline
\end{tabular}

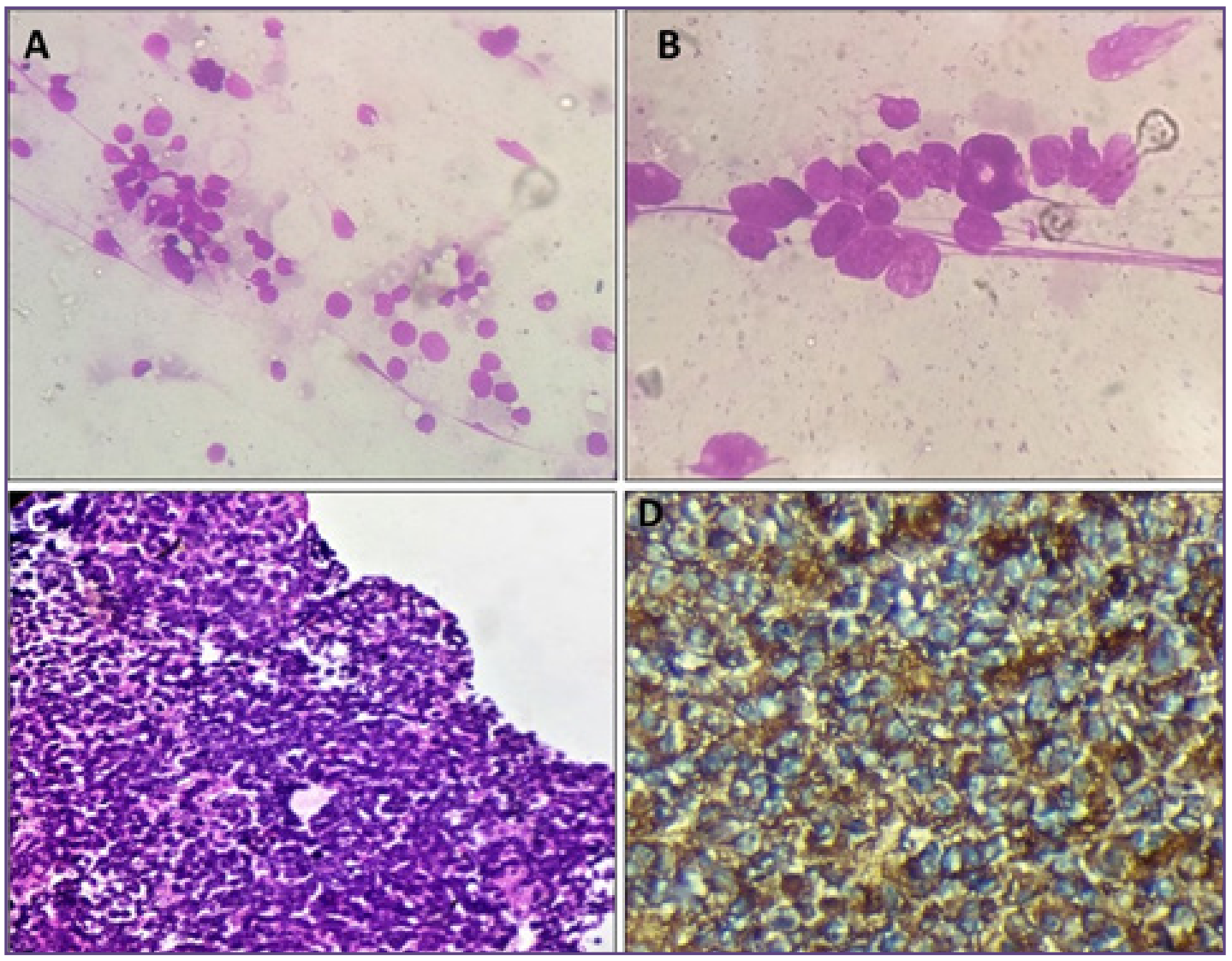

Fig. 1A-D: A\&B-Low \& High-power view of BM aspirate smears from patient with neuroblastoma showing rossette formation of tumor cells (A-Giemsa,200X; B-400X). C\&D-Cellular bone marrow biopsy showing sheets of small blue round cell cells (Fig C-H\&E, 200X) which revealed strong expression of neuron specific enolase (Fig D- NSE, 400X). 


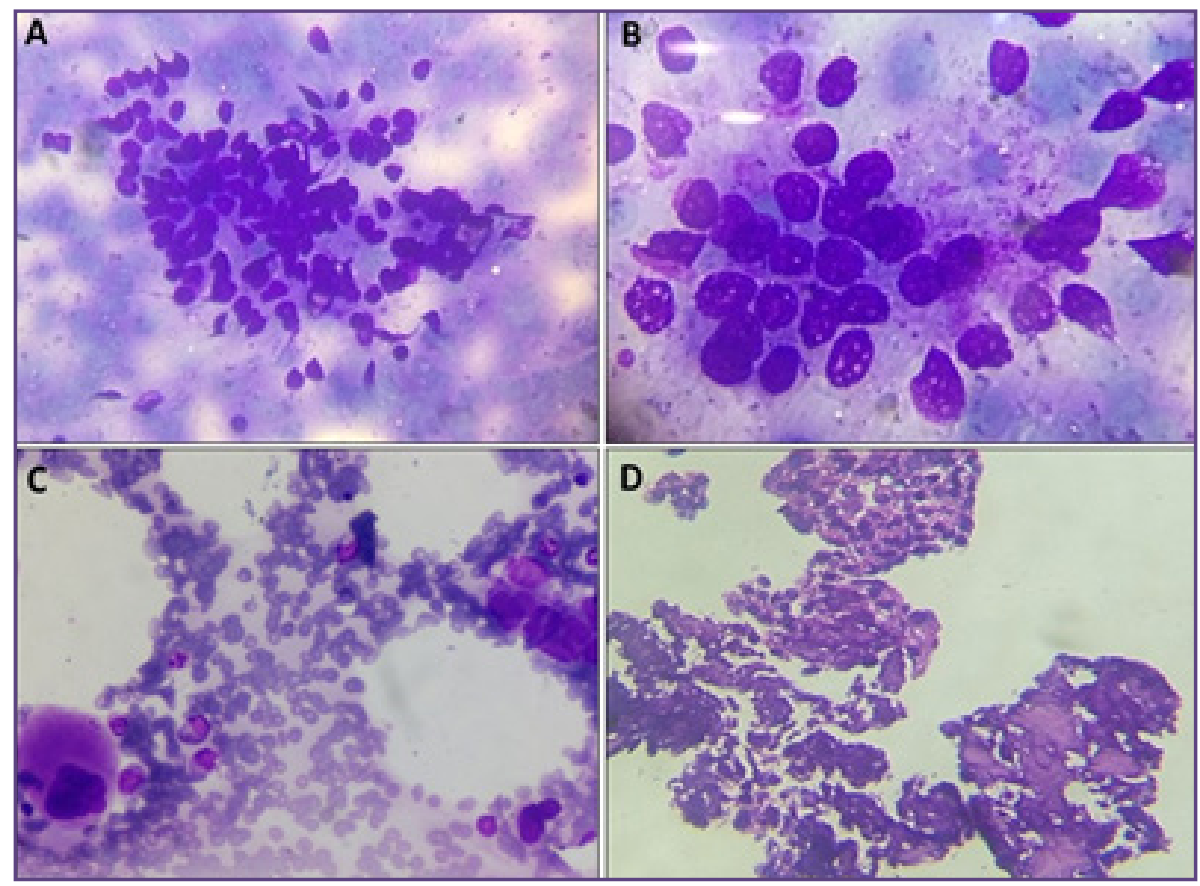

Fig. 2A-D: A-Low power view of BM aspirate smear showing cluster of prostatic adenocarcinoma cells with vague glandular arrangement (Giemsa,200X). B- High power view showing tumor cells with high N/C ratio, hyperchromatic nuclei \& multiple nucleoli (Giemsa,400X). C- A megakaryocyte is noted along with tumor cells and few scattered hematopoietic elements (Giemsa,200X). D-Bone marrow biopsy showing crushing artefact with few sheets of malignant cells from patient with carcinoma prostate $(\mathrm{H} \& \mathrm{E}, 200 \mathrm{X})$.

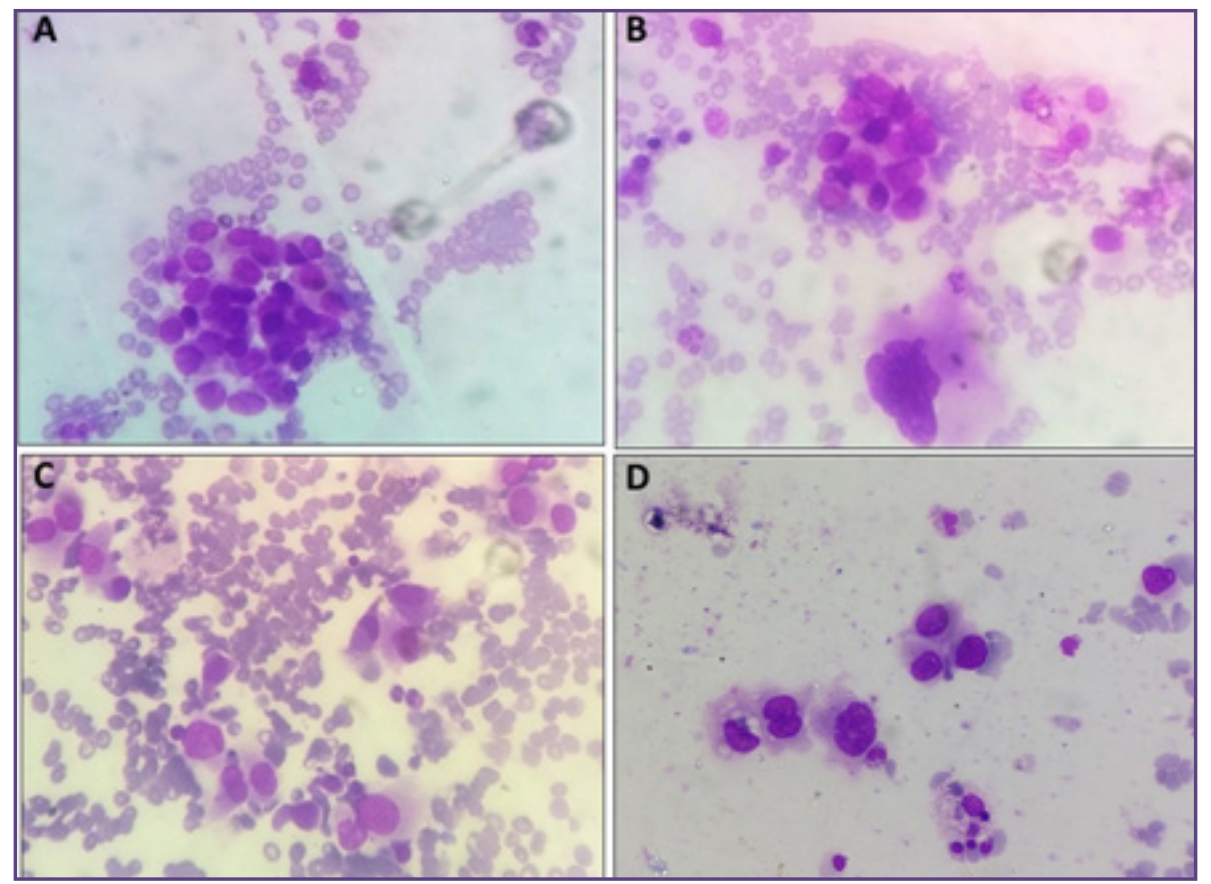

Fig. 3A-D: A- BM aspirate smear from patient with carcinoma breast showing clusters of malignant ductal epithelial cells and scattered hematopoietic elements (Giemsa,200X). B- Figure showing a megakaryocyte with cluster of tumor cell (Giemsa,200X). C\&D: BM smears from patient with hepatic malignancy showing scattered large tumor cells with high N/C ration, hyperchromatic nuclei, moderate amount of cytoplasm (C\&D-Giemsa,200X). 


\section{Discussion}

Primary solid tumors do not usually cause early death of the patient, if treated properly and timely. ${ }^{[1,2]}$ But, once the tumor cells reach and get established in various organs, the mortality of the patient increases markedly. The different tumors have their distinct pattern of dissemination. ${ }^{[2-6]}$ This is due to the anatomical and mechanical structure in the human body which results in the organ preference of tumor metastasis. The communication between the detached tumor cells and distant microenvironment is crucial for the progression of metastatic deposits. ${ }^{[-7]}$

Bone marrow (BM) involvement by metastasis is the most common malignancy of bone, far more than primary bone malignancies. Bone has been placed overall as the third most common site of malignancy after lungs and liver and is the most common site of metastasis from primary breast and prostate carcinomas.

BM contains various cells comprising of hematopoietic origin and cells involved in bone formation and remodelling. The major function of marrow is to regulate hematopoiesis. In the marrow, different cells like osteoblasts, endothelial cells, nerve cells, adipocytes, CXCL-12-abundant reticular cells (CAR cells) and mesenchymal stem cells, all serve as specific niche for the development of hematopoietic stem cells. These cells help the HSCs in maintaining their function like homing, self-renewal, quiescence and differentiation. ${ }^{[3,7]}$

BM metastasis upgrades any solid tumor, hence early detection is the mainstay for treatment. Recent radiological studies have established PET/CT and PET/MRI as sensitive modality for diagnosing bone involvement. ${ }^{[8,9]}$ However, confirmation of the suspected cases requires bone marrow \& histopathological examination including immunohistochemistry (IHC) wherever needed. Moreover, in view of huge cost of these modalities, particularly in developing countries, bone marrow examination is a preferred investigation as it is a simple, rapid, economical yet effective procedure for diagnosis of the disease.

In addition, it is also useful as an important tool for prognostication and to detect an unknown primary in case of a patient presenting with pathological fracture with no detetctable primary lesion [using techniques like IHC and FISH etc]. ${ }^{[3-8]}$ Primary solid tumors are the source of metastatic deposits and hence relapse of the disease process. Henceforth detection of these cells is significant with regard to staging, prognosis and therapeutic decision.

Patients with BM involvement could have normal blood counts, some disturbances in one or more series or they could be pancytopenic. Though severe anemia can be life threatening, the complications of neutropenia and thrombocytopenia result in more morbidity and mortality. Pancytopenic patients are known to have a higher complication risk than the others (Kilickap et al; 2007). ${ }^{[9]}$ In our study, anemia was the most common finding on haematological examination. Metastasis affects the normal haematopoiesis leading to myelopthisic anaemia and other cytopenias.

Leukoerythroblastic blood picture may be noted in onethird to two-third of patients with marrow infiltration. Leukoerythroblastosis usually correlates with extent of fibrosis in marrow not exactly with tumor burden. In our study, 4 of 26 cases (15.4\%) showed this blood picture. However, few studies have reported the frequency as $30-35 \%$. ${ }^{[9-11]}$ Metastases from prostatic carcinoma and neuroblastoma tend to produce generalized infiltration of the marrow and commonly showed leukoerythroblastic anemia, as reported in few of cases in literature. ${ }^{[9-12]}$ In our study also, we reported similar findings with leukoerythoblastic blood picture in 3 cases of prostatic carcinoma and 1 case of SRCT.

Prostate specific antigen (PSA) is also often positive in cases of bone marrow metastases from patients with prostatic carcinoma, even after prostatectomy has been done. In our study we found elevated PSA levels in 6 of 8 cases $(75 \%)$ of prostatic carcinoma patients. In all these patients prostatectomy had been done previously as primary modality of treatment. Comparable results were found by Kaur et al ${ }^{[10]}$ in their study.

In our study, 26 cases showed metastatic BM infiltration by solid tumor after BM examination of 3520 cases. In concordance with previous studies from India and rest of the world, we also found that carcinoma prostate (22\%) was the most common solid tumor to metastatize to bone marrow in adults. ${ }^{[10-15]}$ Metastasis from carcinoma breast and gall bladder/ hepatic case included one case each. In our series, all pediatric cases had infiltration by SRCT.

Unlike indicated in other studies, carcinoma breast and melanoma were not that common to metastatize in the present study. ${ }^{[5,14-18]}$ Tumor cell identification is sometimes a challenging task and needs support of immunohistochemistry as highlighted in one of our cases of SRCT (Neuroblastoma). The initial marrow aspirate in this 11-year-old patient was inadequate and it was difficult to subtype this SRCT. Simultaneously, we received BM biopsy of the patient and put a panel of antibodies like LCA, NSE, CK, CD99, etc. Finally, NSE was found to be diffusely positive confirming the diagnosis of neuroblastoma. 
The presence of detached tumor cells in BM has been correlated with a poor prognosis. The recent published papers on BM metastasis have encouraged more research in order to establish the exact mechanism of cross talk between malignancy and BM microenvironment. ${ }^{[16-20]}$ BM metastasis can lead to disease recurrence even after treatment. So, therapeutic approach to treat BM metastasis is required like HSC mobilizing drug, eg., G-CSF and AMD3100. ${ }^{[8,18-20]}$ These can be utilized for mobilizing the niche-engaged dormant detached tumor cells to re-enter the cell cycle. These metastatic-niche should be the target of various therapeutic interventions. Hence, it is important to understand the exact interaction between the tumor cells and BM "metastatic-niche".

Although BM aspirates give reliable information in majority of the cases regarding presence of metastasis, trephine biopsy along with IHC is pertinent in establishing the definitive diagnosis of BM metastasis particularly in difficult cases.

\section{Conclusion}

$\mathrm{BM}$ is one of the important and infrequent sites of metastasis of solid tumors. Aspiration of the BM provides an easy and quick way of detecting involvement, however, biopsy with IHC may be needed in cases with microscopic tumor burden. BM examination can provide a clue to the primary site in those cases where primary is unknown and metastatic lesions are detected in the BM. BM metastasis is associated with poor prognosis. This study highlights the importance of detection of BM metastasis as it has significant clinical implication with impact on therapeutic decisions.

\section{References}

1. Bussard KM, Gay CV, Mastro AM. The bone microenvironment in metastasis; what is special about bone? Cancer Metastasis Rev 2008;27:41-55.

2. Yu HH, Tsai YY, Hoffe SE. Overview of diagnosis and management of metastatic disease to bone. Cancer Control 2012;19:84-91.

3. Shiozawa Y, Eber MR, Berry JE, Taichman RS. Bone marrow as a metastatic niche for disseminated tumor cells from solid tumors. Bonekey Rep. 2015; 20(4):689.

4. Tasleem RA, Chowdhary ND, Kadri SM, Chowdhary QA. Metastasis of solid tumors in bone marrow: a study from Kashmir India. J Clin Pathol.2003;56:803-804

5. Kołda A, Helbig G, Kopińska A, Wichary R, Pająk J, KyrczKrzemień S, Metastasis of solid tumors into bone marrow -
Single center experience. Acta Haematol Pol(2017), https:// doi.org/10.1016/j.achaem.2017.05.001.

6. Moriwaki SMK, Okabe K, Yamauchi M, Yamamoto T, Kamei T. Pathological analysis for bone marrow metastasis by aspiration and core needle biopsy. Jpn J Cancer Clinic 2003;49(3):203-09

7. L. Demir, M. Akyol, S. Bener et al., "Prognostic evaluation of breast cancer patients with evident bone marrow metastasis," Breast Journal.2014;20(3):279-87.

8. H.-G. Kopp, K. Krauss, T. Fehm et al., "Symptomatic bone marrow involvement in breast cancer-clinical presentation, treatment, and prognosis: a single institution review of 22cases. Anticancer Research. 2011;31(11):4025-30.

9. Kilickap S, Erman M, Dincer M, Aksoy S, Hakan H, Yalcin Y. Bone marrow metastasis of solid tumors: clinicopathological evaluation of 73 cases. Turk J Cancer 2007;37(3):85-8.

10. Kaur G, Basu S, Kaur P, Sood T. Metastatic Bone Marrow Tumors: Study of Nine Cases and Review of the Literature. J Blood Disord Transfus 2011. 2:110. doi:10.4172/2155-9864.1000110.

11. Mohanty SK, Dash S. Bone marrow metastasis in solid tumors. Indian J Pathol Microbiol.2003;46:613-16.

12. Sar R, Aydogdu I, Ozen S, Sevinc A, Buyukberber S. Metastatic bone marrow tumours: a report of six cases and review of the literature. Haematologia. 2001;31:215-23.

13. Kucukzeybek BB, Calli AO, Kucukzeybek Y, Bener S, DereY, et al. The prognostic significance of bone marrow metastasis. :Evaluation of 58 cases. Indian $\mathrm{J}$ Pathol Microbiol.2014 (3):396-399.

14. Saki N, Abroun S, Farshclousti Hagh M, Asgharei F. Neoplastic Bone marrow niche: hematopoietic \& mesenchymal stem cells. Cell J 2011; 13:131-136.

15. Ara T, DeClerckk YA. Interleukin-6 in bone marrow metastasis and cancer progression. Eur J Cancer 2010; 46:1223-1231.

16. Schneider JG, Amend SH, Weilbacecher KM. Integrin \& bone marrow metastasis: integrating tumor cells \& stromal cell interaction. Bone 2011;48:54-65.

17. Rahim F, Haizamani S, Mortaz E, Ahmadzadeh A, Shahjahani M,et al. Molecular regulation of bone marrow metastasis in prostate and breast carcinoma. Bone marrow Res 2014; 2014:405920.

18. Dittus C, Mathew H, Malek A, Negriu A. Bone marrow infiltration as the initial presentation of gastric signet ring cell adenocarcinoma. JGastrointest Oncol 2014;5:113-16.

19. Mahdi EJ, Mahdi AJ. Leukoerythroblastosis and thrombocytopenia as clue to metastatic malignancy. BMJ Case Rep 2014;31:2014

20. Zehentner B. K. Detection of disseminated tumor cells: strategies and diagnostic implications. Expert Rev Mol Diagn 2002;4:41-48. 